\title{
Phase diagram of the system with the repulsive shoulder potential in two dimensions: density functional approach
}

\author{
E. S. Chumakov, ${ }^{1,2}$ Yu.D. Fomin,,${ }^{1,2}$ E. L. Shangina, ${ }^{1}$ E. E. Tareyeva, ${ }^{1}$ E.N. Tsiok, ${ }^{1}$ and V.N. Ryzhov ${ }^{1,2}$ \\ ${ }^{1}$ Institute for High Pressure Physics RAS, 142190 Kaluzhskoe shosse, 14, Troitsk, Moscow, Russia \\ ${ }^{2}$ Moscow Institute of Physics and Technology, 141700 Moscow, Russia
}

(Dated: July 17, 2018)

\begin{abstract}
In the framework of the density functional theory of freezing proposed in our previous works, we calculate the phase diagram of two-dimensional system of particles interacting through the repulsive shoulder potential. This potential consists of the hard core and repulsive shoulder of the larger radius. It is shown that at low densities the system melts through the continuous transition in accordance with the Kosterlitz-Thouless-Halperin-Nelson-Young (KTHNY) scenario, while at high densities the conventional first order transition takes place.

PACS numbers: $61.20 . \mathrm{Gy}, 61.20 . \mathrm{Ne}, 64.60 . \mathrm{Kw}$
\end{abstract}

A large number of papers studying the melting transition in two dimensions have been published during last decades. They include results of real experiments, computer simulations and various theoretical approaches. This is dictated by the growing interest to the behavior of the nanoconfined systems. Confining drastically changes the spatial distribution and the ways of dynamic rearrangement of the molecules in the system. The confined fluids microscopically relax and flow with characteristic times that differ from the bulk fluids. These effects play important role in the thermodynamic behavior of the confined systems and can considerably change the topology of the phase diagram. In general, the motivation for the study of the confined systems follows from the fact that there are a lot of real physical, chemical and biological processes which drastically depend on the properties of such systems [1-6].

It is not surprising that the spatial ordering of molecules depends on the dimensionality of the space to which it is confined. Mermin [7] has shown that in in two dimensions $(2 D)$ the long-range crystalline order can not exist because of the thermal fluctuations and transforms to the quasi-long-range order. On the other hand, the real long range bond orientational order does exist in this case. At high temperatures one can find the conventional isotropic fluid.

The melting scenario in two dimensions is a subject of long lasting controversy. Now it is widely believed that the Kosterlitz, Thouless, Halperin, Nelson, and Young theory (KTHNY theory) 8 11] correctly describes the melting transition in $2 D$. In the framework of the KTHNY theory the two-dimensional melting occurs in the way which is fundamentally different from the melting transition of three-dimensional systems. In $2 D$, the bound dislocation pairs dissociate at some temperature $T_{m}$ transforming the quasi-long range translational order in the short-range, and long-range orientational order into the quasi-long range order. The new phase with the quasi-long range orientational order is called the hexatic phase. After consequent dissociation of the disclination pairs at some temperature $T_{i}$ the system transforms into the isotropic liquid. Both transitions are continuous, in contrast with the conventional first order three dimensional melting.

The unambiguous confirmations of the KTHNY theory have been obtained, for example, from the recent experiments on the colloidal model system with repulsive magnetic dipole-dipole interaction [12 16]. On the other hand, the first-order melting in 2D is also possible [1724]. In Refs. 23, 24] it was shown that at low disclination core energy system can melt through one first-order transition as a result of the dissociation of the disclination quadrupoles.

KTHNY theory is independent on the pair potential of the system and seems universal, however, numerous experimental and simulation studies demonstrate the controversial results: the systems with very short range or hard core potentials melt through weak first-order transition, while the melting scenarios for the soft repulsive particles favor the KTHNY theory [2, 25 50].

In our previous publications the density functional approach for the description of the $2 D$ melting was proposed [19, 20] and it was shown that the hard disk system melts through the first order phase transition, while in the $2 D$ Coulomb system the melting transition occurs in accordance with the KTHNY scenario. In Refs. [21, 22] the density functional calculations were used for the description of the melting transition in the $2 D$ square-well system. It was shown that this system can demonstrate both first-order and continuous melting transitions depending on the width of the attractive well.

In the present paper we extend our previous results to the melting transition in the $2 D$ square-shoulder system in order to study the influence of the width of the shoulder on the phase diagram. The potential is given by the equation:

$$
U(r)= \begin{cases}\infty, & r \leq d \\ \varepsilon, & d<r \leq \sigma . \\ 0, & r>\sigma\end{cases}
$$


where $d$ is the diameter of the hard core, $\sigma$ is the width of the repulsive step, and $\varepsilon$ its height. As it was discussed before 51, 52], in the low-temperature limit $\tilde{T} \equiv k_{B} T / \varepsilon<<1$ the system reduces to a hard-disk system with hard-disk diameter $\sigma$. At the same time, in the limit $\tilde{T}>>1$ the system reduces to a hard-disk model with a smaller hard-disk diameter $d$. In this case, melting at high and low temperatures are described by the simple hard-disk melting curve $P=c T / \sigma^{2}$, where $\sigma^{\prime}$ is the hard-disk diameters ( $\sigma$ and $d$, respectively). A crossover from the low- $T$ to high- $T$ melting behavior takes place for $\tilde{T}=\mathcal{O}(1)$. The precise form of the phase diagram depends on the ratio $s \equiv \sigma / d$. For large enough values of $s$ one should expect to obtain the melting curve with a maximum that should disappear as $s \rightarrow 1$ [51, 52]. In what follows we will use the reduced units $r^{\prime}=r / d, \rho^{\prime}=\rho / d^{2}$ and omit tilde.

The different smoothed versions of the potential (1) (core softened potentials) were discussed recently in order to study the water-like anomalies which appear due to the existence of two length scales in the potential [52 59].

In two dimensions the melting scenarios of the systems with the core softened potentials were studied in Refs. [31 33] in the framework of computer simulations. It was shown, that at low width of the soft core, when the system behaves like an ordinary soft disk system, melting occurs through one weak first order transition. However, with increasing the width of the repulsive shoulder, the phase diagram becomes much more complex. As in Ref. 25], we found that the phase diagram consists of three different crystal phases, one of them with square symmetry and the other two triangular. At low densities, when the soft core of the potential is effective, melting of the triangular phase is a continuous two-stage transition, with an intermediate hexatic phase, in accordance with the KTHNY scenario for this melting transition. At high density part of the phase diagram one finds the square and triangular phases, which melt through one first-order transition. The thermodynamic and dynamic anomalies do exist in this case, however, the order of this anomalies is inverted in comparison with the three-dimensional case [33].

At the same time, in Ref. [4] the phase diagram of a square-shoulder square-well potential was studied in two dimensions. It has been previously shown that this potential exhibits liquid anomalies consistent with a metastable liquid-liquid critical point [60]. It was shown that the melting occurs through the first order transition, despite a small range of metastability.

In order to continue, let us for completeness briefly recall the main ideas of the $2 D$ density functional theory of freezing [19 22]. As it was mentioned above, in $2 D$ the long range translational order can not exist due to the thermal fluctuations. Therefore, at low temperatures the local density of a solid, which is proportional to the one-particle distribution function, can be expanded in a
Fourier series in reciprocal lattice vectors $\mathbf{G}$ :

$$
\rho(\mathbf{r})=\sum_{\mathbf{G}} \rho_{\mathbf{G}}(\mathbf{r}) e^{i \mathbf{G r}},
$$

where $\rho_{\mathbf{G}}(\mathbf{r})$ are the order parameters for the liquid-solid phase transition. Because of the thermal fluctuations, the order parameters $\rho_{\mathbf{G}}(\mathbf{r})$ slowly vary at distances of order $G^{-1}$ and have the amplitude and the phase:

$$
\rho_{\mathbf{G}}(\mathbf{r})=\rho_{\mathbf{G}} e^{i \mathbf{G u}(\mathbf{r})} .
$$

Here $u(\mathbf{r})$ has the meaning of the displacement field, which, in general, can be decomposed into the smooth part corresponding to the phonon field, and singular part, which can be interpreted as the Kosterlitz-Thouless vortices [8] or dislocations.

Taking into account the long range fluctuations, one can write the Landau expansion in the form:

$$
\begin{aligned}
\Delta F & =\frac{1}{2} \int d^{2} r \sum_{\mathbf{G}}\left[A\left|\mathbf{G} \times \nabla \rho_{\mathbf{G}}\right|^{2}+B\left|\mathbf{G} \cdot \nabla \rho_{\mathbf{G}}\right|^{2}+\right. \\
& \left.+\left|\rho_{\mathbf{G}}(\mathbf{G} \cdot \nabla) \rho_{\mathbf{G}}\right|\right]+ \\
& +\frac{1}{2} a_{T} \sum_{\mathbf{G}}\left|\rho_{\mathbf{G}}\right|^{2}+b_{T} \sum_{\mathbf{G}_{1}+\mathbf{G}_{2}+\mathbf{G}_{3}=0} \rho_{\mathbf{G}_{1} \rho_{\mathbf{G}_{2}} \rho_{\mathbf{G}_{3}}+} \\
& +O\left(\rho^{4}\right) .
\end{aligned}
$$

$\Delta F$ corresponds to the difference of the free energy of crystal and isotropic liquid. The first term in the expansion (4) has the form of the free energy of a deformed solid. The Lame coefficient $\mu$ is a function of the parameters $A, B$, and $C$ and is proportional to the squared modulus of the order parameter (2).

With the help of equation (4), the $2 D$ melting scenario can be described in the following way. First of all, one can neglect the fluctuations of the order parameter (like in three dimensions). In this case, from Eq. (4) one can see that there is a possibility of the ordinary transition when at some temperature $T_{M F}$ the modulus of the order parameter becomes zero. Because of the third-order term in the Landau expansion (44), the transition is of the first order. However, there is another possibility: at temperature $T_{m}$ the singular fluctuations of the phase of the order parameter (vortices), which corresponds to the free dislocations, appear in accordance with the standard Kostelitz-Thouless paradigm. In this case the modulus of the order parameter is not zero, however, the system will respond to shear stress with no resistance $(\mu=0)$, and it is therefore possible to call the phase above $T_{m}$ as a liquid. Really this is a hexatic phase with the quasi-long range orientational order [9-11]. There are two possibilities: (i) $T_{m}<T_{M F}$, and the system melts through the continuous Kosterlitz-Thouless transition; (ii) $T_{m}>T_{M F}$, the system melts through the first-order transition.

$T_{m}$ and $T_{M F}$ can be calculated using the microscopic expressions for the (Helmholtz) free energy $F$ of the solid 
and for the elastic moduli. These expressions may be obtained in the framework of the density functional theory of freezing [19 22]. In this case the local density of the solid, $\rho(\mathbf{r})$, can be represented as localized Gaussians (of width $1 / \alpha^{1 / 2}$ ) at lattice sites, i.e., with Fourier components $\rho_{\mathbf{G}}=\rho \exp \left(-G^{2} / 4 \alpha\right)$, where $\rho=\int d \mathbf{r} \rho(\mathbf{r}) / V$ is the average density. Because our main purpose is to obtain a qualitative description of the melting transition in the system with the repulsive step potential, we use the simplest but correct enough version of the density functional theory [19 22, 61, 62]:

$$
\begin{aligned}
& \beta \Delta F=\int d \mathbf{r} \rho(\mathbf{r}) \ln [\rho(\mathbf{r}) / \rho]- \\
& -\frac{1}{2} \int d \mathbf{r} \int d \mathbf{r}^{\prime} c^{(2)}\left(\left|\mathbf{r}-\mathbf{r}^{\prime}\right|, \rho\right)[\rho(\mathbf{r})-\rho]\left[\rho\left(\mathbf{r}^{\prime}\right)-\rho\right],
\end{aligned}
$$

where $c^{(2)}\left(\left|\mathbf{r}-\mathbf{r}^{\prime}\right|, \rho\right)$ is the direct correlation function [63], $\beta=1 / k_{B} T$. The localization parameter $\alpha$ is fixed by minimizing the total free energy with respect to it. In principle, the parameters of the first order melting transition should be determined from Eq. (6) using the double tangent construction, however, for the approximate qualitative description of the phase diagram it seems sufficient to apply the equation $\Delta F=0$ in order to determine $T_{M F}$.

The temperature $T_{m}$ can be determined from the KTHNY criterion [9 11] which determines the instability of the crystal lattice with respect to the appearance of free dislocations:

$$
K\left(T_{m}\right)=\frac{a_{0}^{2}}{k_{B} T_{m}} \frac{4 \mu\left(T_{m}\right)\left(\mu\left(T_{m}\right)+\lambda\left(T_{m}\right)\right)}{2 \mu\left(T_{m}\right)+\lambda\left(T_{m}\right)}=16 \pi,
$$

where $\mu(T)$ and $\lambda(T)$ are the Lame coefficients and $a_{0}$ is the lattice constant for the triangular lattice: $a_{0}^{2}=$ $2 /(\sqrt{3} \rho)$.

Expressions for the Lame coefficients have the form [21, 22]:

$$
\begin{aligned}
\mu & =\frac{k_{B} T}{16 \rho} \sum_{G} \rho_{G}^{2} m_{G} G^{2}\left(\gamma_{G}+2 \delta_{G}\right), \\
\lambda & =\frac{k_{B} T}{16 \rho} \sum_{G} \rho_{G}^{2} m_{G} G^{2}\left(\gamma_{G}-6 \delta_{G}\right)+ \\
& +k_{B} T \rho\left(1-\rho \tilde{c}^{(2)}(0)\right),
\end{aligned}
$$

where

$$
\begin{aligned}
& \gamma_{G}=2 \pi \rho \int r^{3} d r c^{(2)}(r ; \rho) J_{0}(G r), \\
& \delta_{G}=2 \pi \rho \int r^{3} d r c^{(2)}(r ; \rho) J_{1}(G r) /(G r),
\end{aligned}
$$

and $J_{0}(x)$ and $J_{1}(x)$ are the Bessel functions, $m_{G}$ is the number of reciprocal vectors with the same length, and $\tilde{c}^{(2)}(q)$ is the Fourier transform of the direct correlation function.
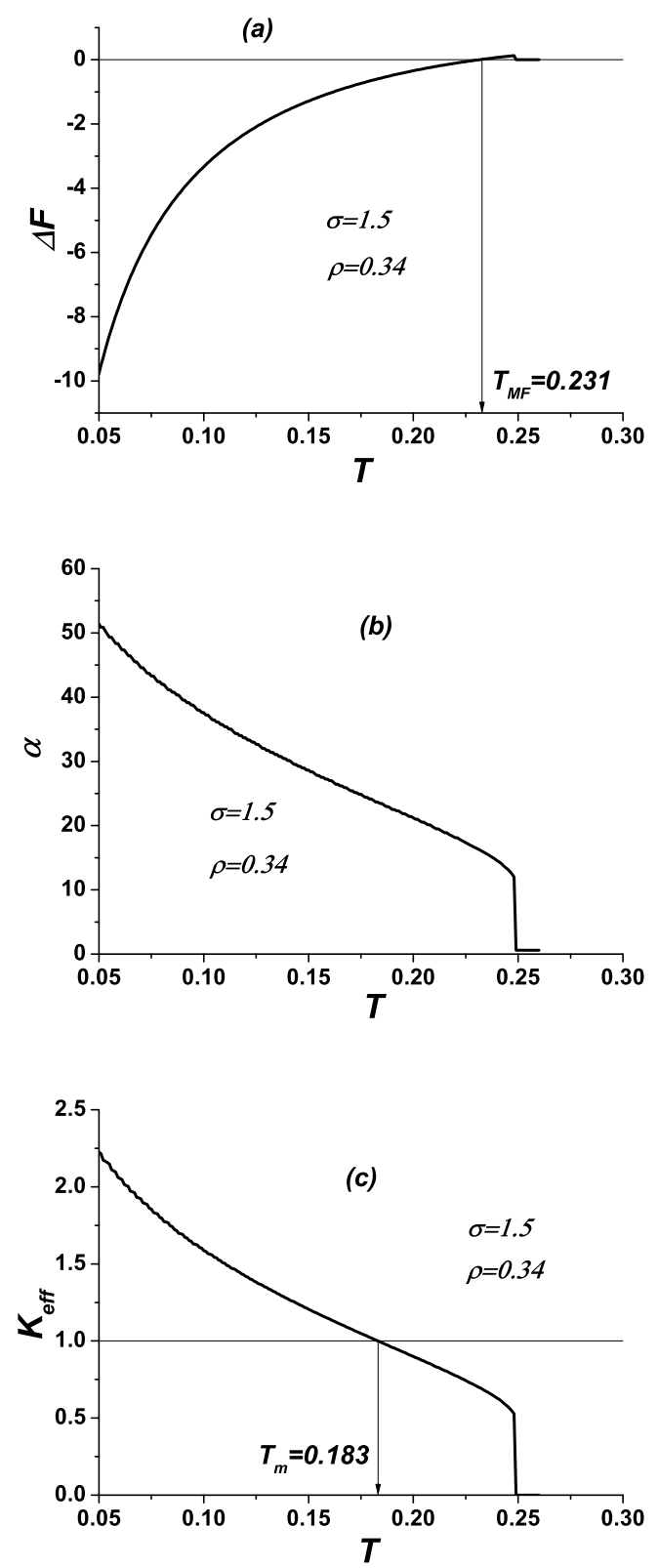

FIG. 1: $\Delta F(\mathrm{a}), \alpha(\mathrm{b})$, and $K_{\text {eff }}=K / 16 \pi$ (c) as functions of temperature for $\rho=0.34$.

For the further calculations one needs an approximate expression for the direct correlation function. We use the simple approximation for the direct correlation function 63] of the hard-core system, suggested by Lovett 64] (see also [21, 22, 65, 66]):

$$
c^{(2)}(r, \rho)=\left\{\begin{array}{ll}
c_{H D}^{(2)}(r, \rho), & r \leq d \\
-\frac{\phi(r)}{k_{B} T}, & r>d
\end{array},\right.
$$

where $c_{H D}^{(2)}(r, \rho)$ is the hard disks direct correlation function and $\phi(r)$ is the repulsive shoulder or attractive part 

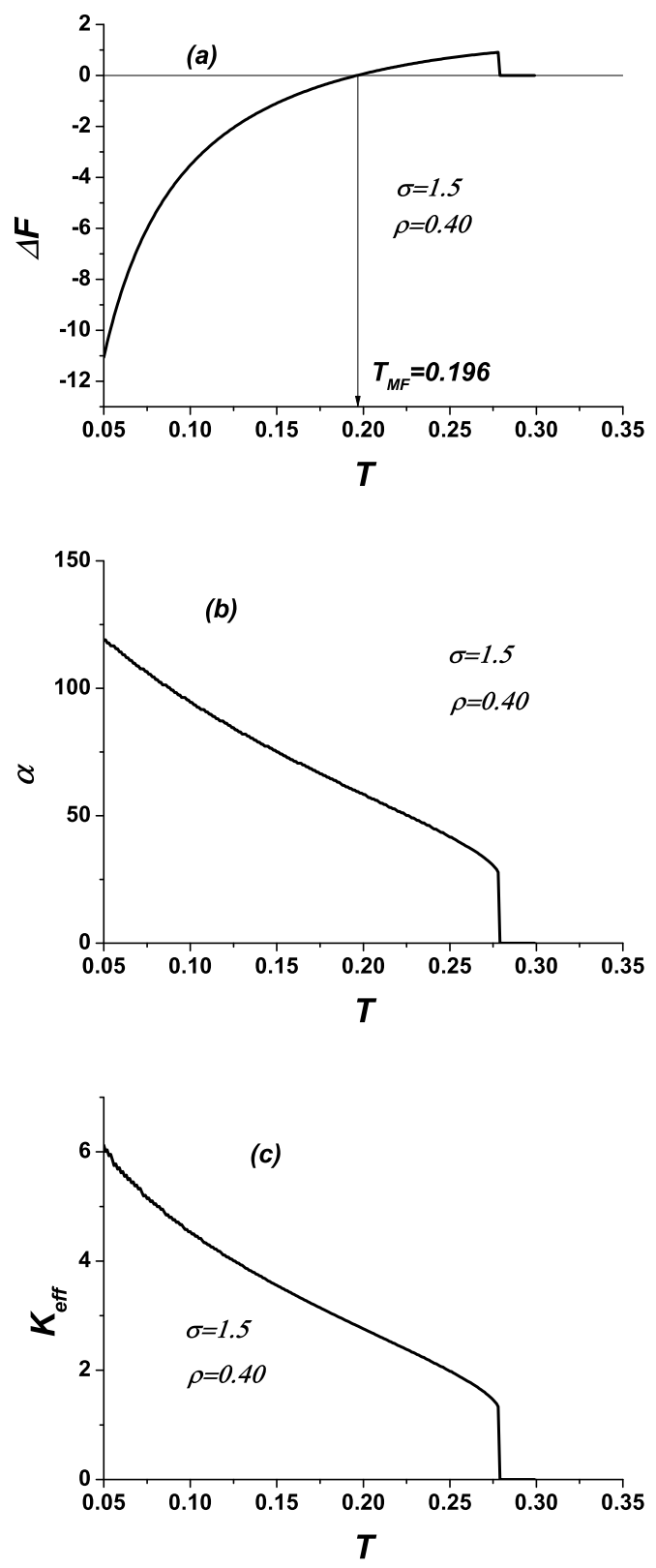

FIG. 2: $\Delta F(\mathrm{a}), \alpha(\mathrm{b})$, and $K_{\text {eff }}=K / 16 \pi(\mathrm{c})$ as functions of temperature for $\rho=0.40$.

of the potential. This approximation should be a good one when $-\frac{\phi(r)}{k_{R} T}$ is small. The approximation, though rough, is similar in spirit to the mean spherical model approximation which has been found to be a good approximation in many cases [63]. In the case of the potential (11), Eq. (9) takes the form:

$$
c^{(2)}(r, \rho) \approx\left\{\begin{array}{ll}
c_{H D}^{(2)}(r, \rho), & r \leq d \\
\frac{-\varepsilon}{k_{B} T}, & d<r \leq h . \\
0, & r>h
\end{array} .\right.
$$

For $c_{H D}^{(2)}(r, \rho)$ we use the approximate analytic equa-
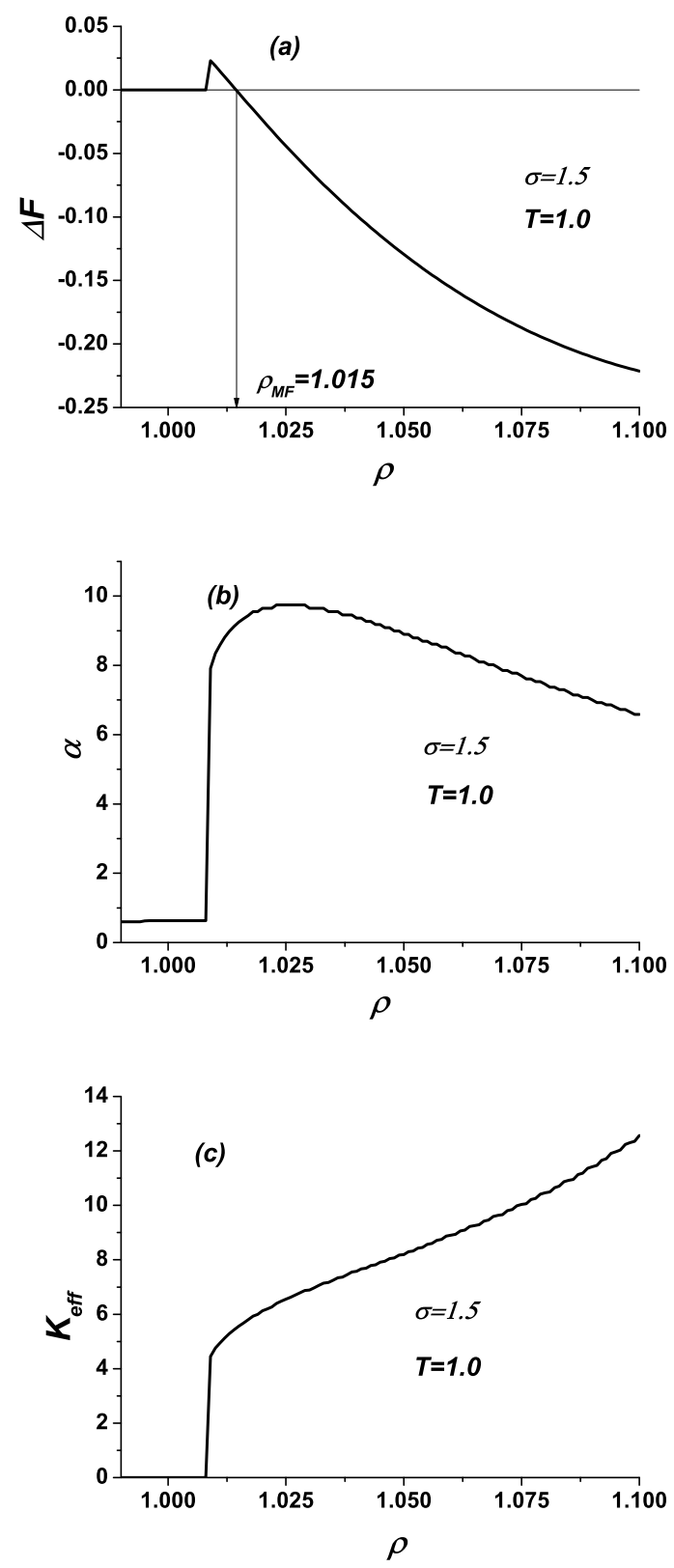

FIG. 3: $\Delta F(\mathrm{a}), \alpha(\mathrm{b})$, and $K_{\text {eff }}=K / 16 \pi$ (c) as functions of density for $T=1.0$.

tion obtained in Refs. 67, 68]:

$$
\begin{aligned}
& c_{H D}^{(2)}(x ; \eta)=-\left[\frac{\partial}{\partial \eta}(\eta Z(\eta))\right] \Theta(1-x) \times \\
& \times\left\{1-a^{2} \eta+\frac{2}{\pi} a^{2} \eta\left[\arccos \frac{x}{a}-\frac{x}{a}\left(1-\frac{x^{2}}{a^{2}}\right)^{1 / 2}\right]\right\}, \\
& Z(\eta)=\left(1+c_{2} \eta^{2}\right) /(1-\eta)^{2}, \\
& a=\left(2+\eta \alpha_{2}(\eta)\right) /\left(1+\eta+\eta \alpha_{2}(\eta)\right),
\end{aligned}
$$

where $c_{2}=0.128 ; \alpha_{2}(\eta)=-0.2836+0.2733 \eta ; \eta=$ $\pi \rho d^{2} / 4$. 


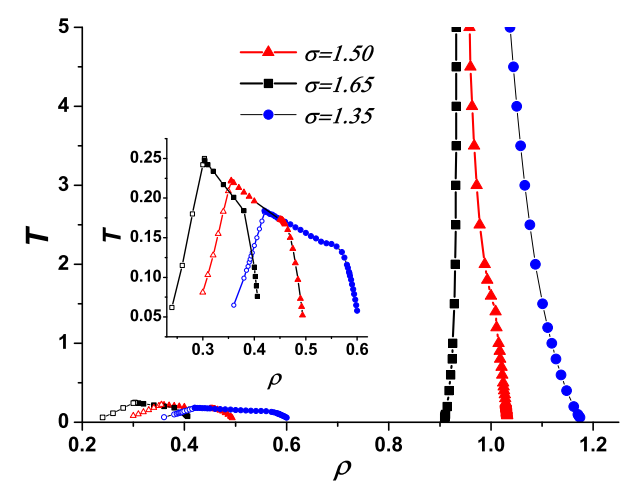

FIG. 4: Phase diagram of the system with the potential (1) for three repulsive shoulder widths: $\sigma=1.50$ (triangles), $\sigma=$ 1.35 (circles), $\sigma=1.65$ (squares). Open symbols correspond to the continuous transitions, while the bold symbols mark the first order transitions.

In Figs. 1 and 2 we represent the behavior of $\Delta F$ (see Eq. (4)), localization parameter $\alpha$, and $K_{\text {eff }}=$ $K(T) / 16 \pi$ (Eq. (6) ) as a function of $T$ for $\sigma=1.5$ and $\rho=0.34$ (Fig. 1) and $\rho=0.4$ (Fig. 2). One can see, that for $\rho=0.34$ the solution of equation $\Delta F=0$ which determines the first-order transition temperature $T_{M F}$, is $T_{M F}=0.231$ (Fig. 1(a)), while the solution of the equation $K_{\text {eff }}=1$ (Fig. 1(c)) $T_{m}=0.183<T_{M F}$. As it was discussed above, in this case the melting should occur in accordance with the KTHNY scenario. From Fig. 1(b) one can conclude that the localization parameter $\alpha$ is well defined till the limit of metastability of the crystal lattice $T_{m e t}=0.248$. On the other hand, for $\rho=0.40$ the situation is different. In this case $T_{M F}=0.196$ while the equation $K_{\text {eff }}=1$ does not have solution till the limit of metastability $T_{m e t}=0.278$. In this case melting occurs through the first order transition.

At high densities there is only weak dependence of the melting density on temperature (see below). In this case, in calculations it is more convenient to fix the temperature and change the density. The typical results are shown in Fig. 3 for $\sigma=1.5$ and $T=1.0$. One can see that at high densities for $T=1.0$ there is the first order melting transition at $\rho_{M F}=1.015$. The equation $K_{\text {eff }}=1$ does not have solution till the limit of metastability $\rho_{\text {met }}=1.009$.

In Fig. 4 we present the resulting phase diagram for three widths of the repulsive shoulder in the potential (10): $\sigma=1.50, \sigma=1.35$, and $\sigma=1.65$. In accordance with the qualitative discussion after Eq. (10), the phase diagram consists in two parts - the low density triangle lattice with the maximum on the melting curve and the high density triangle lattice. It is interesting that at lowest density part of the phase diagram the KTHNY scenario takes place, while with the increasing density the melting becomes the first order transition. Taking into account the fact, that with increasing the density the hard core of the potential (11) becomes effective, one can conclude that this result is consistent with the mentioned above possibility that the systems with soft potentials probably melt in accordance with the KTHNY scenario, while the hard core systems melt through the first order transition. It should be noted that with increasing temperature the system has to behave more and more closely to the hard disk system. There are different estimates for the melting density of hard disk systems [19, 20, 35, 36, 38] which vary from $\rho=0.905$ to $\rho=0.933$. One can see that despite very simple approximations, the results presented in Fig. (4for high temperatures, are in good enough qualitative agreement with previous estimates.

We also considered the possible square lattice but found that it is less stable than the triangle lattice for all densities.

It should be noted that the similar behavior was found in computer simulation of the smoothed version of the potential (11) 31 33]. As it was mentioned in the introduction, in Refs. 31-33] it was shown that at low densities melting occurs through two continuous transitions with the intermediate hexatic phase, however, at high densities only first order transition takes place. As in the present case, the "gap" between the two parts of the phase diagram takes place which increases with increasing the width of the repulsive shoulder. In principle, some crystal lattice can exists in this range of densities at low enough temperatures, however, we could not find it in the present work. Another open question exists for further study. It is related with the crossover from the continuous to first order transition in the phase diagram in Fig. 4. It must be the tricritical point on the melting line, however, in the present study we could give only rough enough estimate for the location of this point without investigation of the properties of this point.

In conclusion, in the present study we consider the melting transition of the repulsive shoulder potential system (11). In the framework of the density functional theory of freezing we calculate the phase diagram and show that it consists of two parts with different melting scenarios (see Fig. 4). At low densities the system melts through the continuous transition in accordance with the Kosterlitz-Thouless-Halperin-NelsonYoung (KTHNY) scenario, while at high densities the conventional first order transition takes place. Taking into account the fact, that with increasing the density the hard core of the potential (10) becomes effective, one can conclude that this result is consistent with the possibility that the systems with soft potentials probably melt in accordance with the KTHNY scenario, while the hard core systems melt through the first order transition.

The authors are grateful to S.M. Stishov and V.V. Brazhkin for valuable discussions. The work was sup- 
ported by the Russian Science Foundation (Grant No 14-22-00820).

[1] M. Alcoutlabi and G. B. McKenna, J. Phys.: Condens. Matter 17, R461 (2005).

[2] S.A. Rice, Chem. Phys. Lett. 479, 1 (2009).

[3] L.B. Krott and M.C. Barbosa, J. Chem. Phys., 138 084505 (2013).

[4] A.M. Almudallal, S.V. Buldyrev, and I. Saika-Voivod, J. Chem. Phys., 137034507 (2012).

[5] L.B. Krott and J.R. Bordinb, J. Chem. Phys. 139, 154502 (2013).

[6] L. B. Krott and M. C. Barbosa, Phys. Rev. E 89, 012110 (2014).

[7] N. D. Mermin, Phys. Rev. 176, 250 (1968).

[8] M. Kosterlitz and D.J. Thouless, J. Phys. C 61181 (1973).

[9] B.I. Halperin and D.R.Nelson, Phys. Rev. Lett. 41121 (1978).

[10] D.R.Nelson and B.I. Halperin, Phys. Rev. B 192457 (1979).

[11] A.P. Young, Phys. Rev. B 191855 (1979).

[12] Urs Gasser, C. Eisenmann, G. Maret, and P. Keim, ChemPhysChem 11, 963 (2010).

[13] K. Zahn and G. Maret, Phys. Rev. Lett. 853656 (2000).

[14] P. Keim, G. Maret, and H.H. von Grunberg, Phys. Rev. E 75, 031402 (2007).

[15] S. Deutschlander, T. Horn, H. Lowen, G. Maret, and P. Keim, Phys. Rev. Lett. 11, 098301 (2013).

[16] T. Horn, S. Deutschlander, H. Lowen, G. Maret, and P. Keim, Phys. Rev. E 88, 062305 (2013).

[17] S.T. Chui, Phys. Rev. B 28, 178 (1983).

[18] W. Janke and H. Kleinert, Phys. Rev. B 41, 6848 (1990).

[19] V.N. Ryzhov and E.E. Tareyeva, Phys. Rev. B 518789 (1995).

[20] V.N. Ryzhov and E.E. Tareeva, Zh. Eksp. Teor. Fiz. 108, 2044 (1995) [J. Exp. Theor. Phys. 81, 1115 (1995)].

[21] V.N. Ryzhov and E.E. Tareyeva, Physica A 314, 396 (2002).

[22] V.N. Ryzhov and E.E. Tareyeva, Theor. Math. Phys. 130, 101 (2002)(DOI: 10.1023/A:1013884616321).

[23] V.N. Ryzhov, Theor. Math. Phys. 88, 990 (1991) (DOI: 10.1007/BF01027701).

[24] V.N. Ryzhov, Zh. Eksp. Teor. Fiz. 100, 1627 (1991) [Sov. Phys. JETP 73, 899 (1991)].

[25] S. Prestipino, F. Saija, and P.V. Giaquinta, J. Chem. Phys. 137, 104503 (2012).

[26] P. Bladon and D. Frenkel, Phys. Rev. Lett. 74, 2519 (1995).

[27] S.I. Lee and S.J. Lee, Phys. Rev. E 78, 041504 (2008).

[28] S. Prestipino, F. Saija, and P.V. Giaquinta, Phys. Rev. Lett. 106, 235701 (2011).

[29] R. Zangi and S. A. Rice, Phys. Rev. E 58, 7529 (1998).

[30] D. Frydel and S. A. Rice, Phys. Rev. E 68061405 (2003).

[31] D.E. Dudalov, Yu.D. Fomin, E.N. Tsiok, and V.N. Ryzhov, J. Phys.: Conference Series 510, 012016 (2014) (doi:10.1088/1742-6596/510/1/012016).

[32] D.E. Dudalov, Yu.D. Fomin, E.N. Tsiok, and V.N. Ryzhov, J. Chem. Phys. 141, 18C522 (2014).

[33] D.E. Dudalov, Yu.D. Fomin, E.N. Tsiok, and V.N.
Ryzhov, Soft Matter 10, 4966 (2014).

[34] J. Lee and K.J. Strandburg, Phys. Rev. B 46, 11190 (1992).

[35] H. Weber, D. Marx, and K. Binder, Phys. Rev. B 51, 14636 (1995).

[36] C.H. Mak, Phys. Rev. E 73, 065104 (2006).

[37] A. Jaster, Europhys. Lett., 42, 277 (1998).

[38] A. Jaster, Phys. Lett. A 330, 120 (2004).

[39] K. Bagchi, H.C. Andersen, and W. Swope, Phys. Rev. Lett. 76, 255 (1996).

[40] K. Bagchi, H.C. Andersen, and W. Swope, Phys. Rev. E 53, 3794 (1996).

[41] E.P. Bernard and W. Krauth, Phys. Rev. Lett. 107, 155704 (2011).

[42] M. Engel, J.A. Anderson, S.C. Glotzer, M. Isobe, E.P. Bernard, and W. Krauth, Phys. Rev. E 87, 042134 (2013).

[43] K. Binder, S. Sengupta, and P. Nielaba, J. Phys.: Condens. Matter 14, 2323 (2002).

[44] R. K. Kalia and P. Vashishta, J. Phys. C: Solid State Phys., 14, L643 (1981).

[45] J. Q. Broughton, G. H. Gilmer, and J. D. Weeks, Phys. Rev. B 25, 4651 (1982).

[46] R. S. Singh, M. Santra, and B. Bagchi, J. Chem. Phys. 138, 184507 (2013).

[47] Keola Wierschem and Efstratios Manousakis, Phys. Rev. B 83, 214108 (2011).

[48] N. Gribova, A. Arnold, T. Schilling, and C. Holm, J. Chem. Phys. 135, 054514 (2011).

[49] Yu. E. Lozovik and V. M. Farztdinov, Solid State Commun. 54, 725 (1985).

[50] Yu. E. Lozovik, V. M. Farztdinov, B. Abdullaev and S. A. Kucherov, Phys. Lett. 112A, 61 (1985).

[51] S. M. Stishov, Phil. Mag. B 82, 1287 (2002).

[52] Y.D. Fomin, N.V. Gribova, V.N. Ryzhov, S.M Stishov, and D. Frenkel, J. Chem. Phys. 129, 064512 (2008).

[53] S.V. Buldyrev, G. Malescio, C.A. Angell, N. Giovambattista, S. Prestipino, F. Saija, H.E. Stanley, and L. Xu, J. Phys.: Condens. Matter 21, 504106 (2009).

[54] P. Vilaseca and G. Franzese, Journal of Non-Crystalline Solids 357, 419 (2011).

[55] N.V. Gribova, Y.D. Fomin, D. Frenkel, and V.N. Ryzhov, Phys. Rev. E 79051202 (2009).

[56] Y.D. Fomin, E.N. Tsiok, and V.N. Ryzhov, J. Chem. Phys. 135, 234502 (2011).

[57] Y.D. Fomin, E.N. Tsiok, and V.N. Ryzhov, J. Chem. Phys. 135, 124512 (2011).

[58] R.E. Ryltsev, N.M. Chtchelkatchev, and V.N. Ryzhov, Phys. Rev. Lett. 110, 025701 (2013).

[59] Y.D. Fomin, E.N. Tsiok, and V.N. Ryzhov, Phys. Rev. E 87, 042122 (2013).

[60] M.R. Sadr-Lahijany, A. Scala, S.V. Buldyrev, H.E. Stanley, Phys. Rev. Lett. 81, 4895 (1998).

[61] M. Baus, J. Phys.: Condens. Matter 2, 2111 (1990).

[62] Y. Singh, Phys. Rep. 207, 351 (1991).

[63] J. P. Hansen, I. R. McDonald, Theory of simple liquids (Academic Press, 1986).

[64] R. Lovett, J. Chem. Phys. 66, 1225 (1977).

[65] V.N. Ryzhov, E.E. Tareyeva, and Yu.D. Fomin, Theor. Math. Phys. 167, 645 (2011).

[66] V. V. Brazhkin, Yu. D. Fomin, V. N. Ryzhov, E. E. Tareyeva, and E. N. Tsiok, Phys. Rev. E 89, 042136 (2014).

[67] J.L. Colot, M. Baus, Phys. Lett. A 119, 135 (1986). 
[68] M. Baus, J.L. Colot, Phys. Rev. A 36, 3912 (1987). 


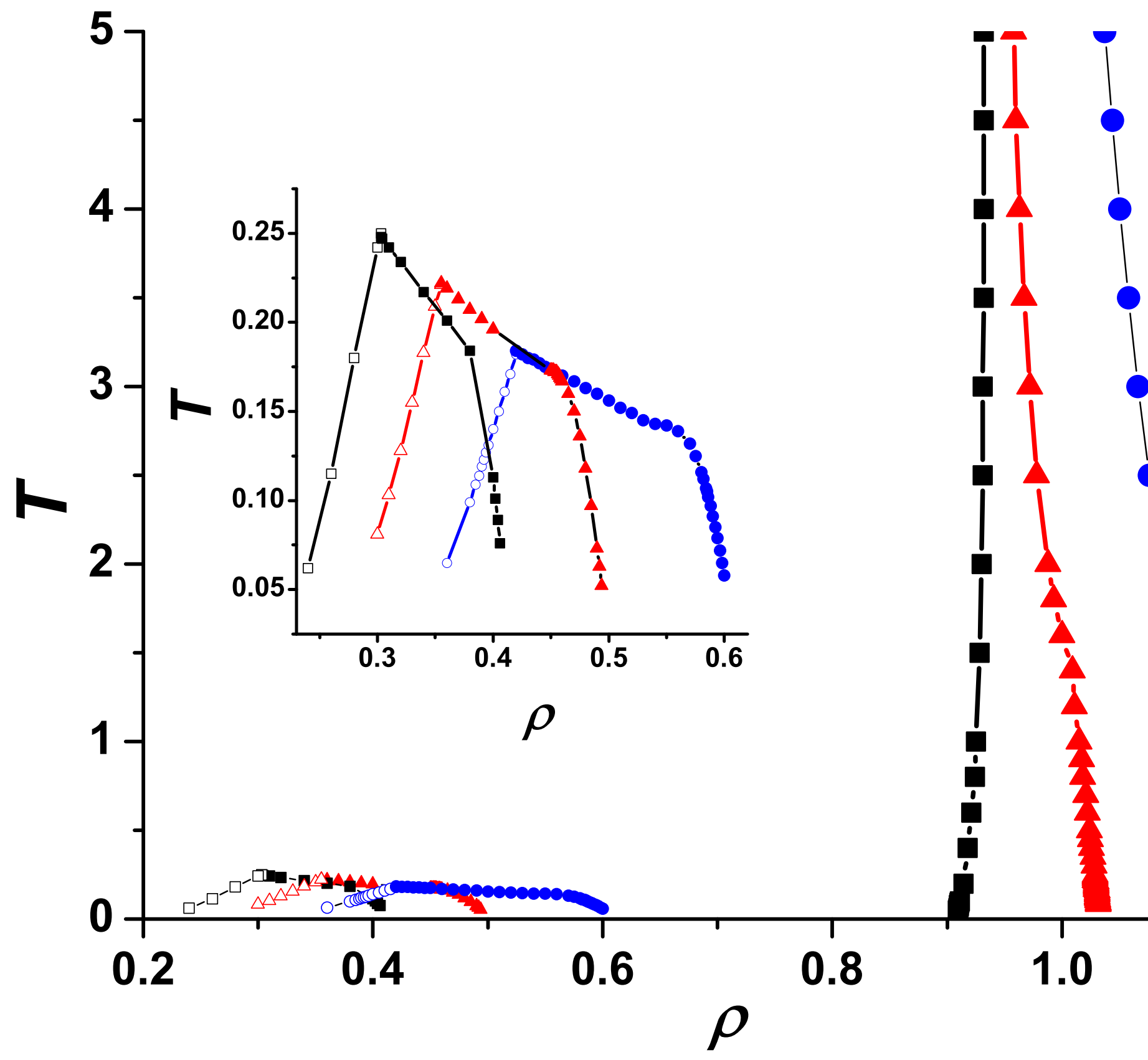

